\title{
Value of IPACK Block (Interspace between the Popliteal Artery and the Capsule of the Posterior Knee) with Adductor Canal Block in Total Knee Arthroplasty
}

\author{
Mohamed Abd-Allah Amin, ${ }^{1}$ MD, Usama I Abotaleb, ${ }^{2}$ MD.
}

\section{* Corresponding Author: \\ Mohamed Abd-Allah Amin drmaa73@gmail.com}

\section{Received for publication December 28, 2020; Accepted February 16, 2021; Published online February 16, 2021.}

Copyright 2021 The Authors
published by Al-Azhar
University, Faculty of Medicine,
Cairo, Egypt. All rights reserved.
This an open-access article
distributed under the legal terms,
where it is permissible to
download and share the work
provided it is properly cited. The
work cannot be changed in any
way or used commercially.
doi: 10.21608/aimj.2021.55090.1381.
${ }^{1}$ Anesthesia and Intensive care
Department, Faculty of Medicine
(Girls), Al-Azhar University.
${ }^{2}$ Anesthesia and Intensive care
Department, Faculty of Medicine,
Al-Azhar University.

\begin{abstract}
Background: Acute postoperative pain after total knee arthroplasty (TKA) is so severe to the degree that necessitates proper analgesia which is one of the patients' human rights and prevents the drawbacks of pain on various body systems. Local anesthetic Infiltration between the Popliteal Artery and the Capsule of the Knee (IPACK) can represent a promising technique for management of postoperative pain in combination with adductor canal block (ACB) after TKA.

Aim of the study: aim is to evaluate the efficiency of IPACK in combination with ACB in relieving postoperative pain after TKR, particularly posterior knee pain compared to ACB alone.

Patients and Methods: Patients received an ACB (Group I) or ACB plus IPACK (Group II) as a component of a multimodal analgesic for TKA. Visual analogue scale (VAS) for postoperative pain assessment were assessed as the primary outcome and opioid consumption, time to first rescue analgesia, and patient satisfaction were assessed as secondary outcomes.

Results: Regarding VAS, there were nonsignificant differences between both groups in the first $8 \mathrm{hrs}$ postoperative, while after $8 \mathrm{hrs}$ postoperative and up to $48 \mathrm{hr}$ postoperative, VAS scores were lower in Group II. Total morphine consumption was less in Group II which had a longer time to first rescue analgesia than Group I. Patient satisfaction 48hr postoperative was higher in Group II than in Group I.

Conclusion: The combination of IPACK block with ACB has the potential of being an adequate technique for management of acute postoperative pain after TKA but this needs more researches of larger samples and use of other types of local anesthetics with different volumes and concentrations.
\end{abstract}

Keywords: Adductor canal block; IPACK; total knee arthroplasty; acute postoperative

pain.

Disclosure: The authors have no financial interest to declare in relation to the content of this article. The Article Processing Charge was paid for by the authors.

Authorship: All authors have a substantial contribution to the article.

\section{INTRODUCTION}

Acute postoperative pain after total knee arthroplasty (TKA) is so severe to the degree that necessitates proper analgesia which is one of the patients' human rights and prevents the drawbacks of pain on various body systems. Additionally, improper pain management leads to prolongation of hospital stay and rehabilitation period with the possibility of progression of acute pain to chronic one ${ }^{1}$. About $60 \%$ of patients subjected to TKA had severe postoperative knee pain and about $30 \%$ of them experienced moderate pain. This high incidence of pain may push some patients to take the decision of not to be subjected to TKA ${ }^{2,3}$.

Non-opioid analgesics alone are not enough to achieve the targeted pain relieve after this type of surgery and opioids alone, usually are not given in the optimal doses for fear of its side effects which include nausea,

vomiting, respiratory depression and possibility of addiction $^{3}$.

Multimodal analgesia is an ideal method for management of acute pain after TKA which may include preemptive analgesia, neuroaxial blockade, peripheral nerve block, and both narcotic and nonnarcotic analgesics. One of the peripheral nerve blocks used for analgesia after TKA is adductor canal block (ACB) and this block may affect the vastus medialis muscle as its supplying nerve is one of the contents of the adductor canal. Another example of these nerve blocks is femoral nerve block (FNB) which may affect the motor function of quadriceps muscle $^{4,5}$. Also, both ACB and FNB may be 
associated with posterior knee pain that necessitates additional analgesics ${ }^{6}$. If sciatic nerve block is performed with femoral nerve block (FNB) for better analgesia, it may increase the risk of fall due to distal motor block ${ }^{7}$.

An interesting nerve block technique described by Sanjay K. Sinha, is an ultrasound-guided local anesthetic Infiltration between the Popliteal Artery and the Capsule of the Knee (IPACK) aiming at blockade of sciatic nerve terminal branches for analgesia after TKA without motor affection ${ }^{\mathbf{8}}$.

The aim of this prospective study is to evaluate the efficiency of IPACK in combination with ACB in relieving postoperative pain after TKA, particularly posterior knee pain compared to ACB alone considering that this technique is relatively new and there are not much studies about evaluating this technique for this purpose.

The primary outcome measure was the visual analogue scale (VAS) for postoperative pain assessment. Secondary outcome measures included total morphine consumption during postoperative 48 hours, time to first rescue analgesia, and patient satisfaction.

\section{PATIENTS AND METHODS}

This prospective double blinded randomized controlled study was conducted from September 2018 to March 2020 in Al-Azhar university hospitals. After obtaining the institutional approval and patients' informed consents, 60 patients enrolled in the study and randomly assigned by computergenerated random number tables to one of two equal groups: Group I (control group) received adductor canal block (ACB) while Group II (IPACK group) received ACB plus local anesthetic Infiltration between the Popliteal Artery and the Capsule of the Knee (IPACK block).

Patients involved in the study were ASA physical status I-III, 21-65 years old, and subjected to unilateral total knee arthroplasty (TKA). Exclusion criteria included patient refusal, neuromuscular disorders, allergy to local anesthetics, and contraindications to spinal anesthesia as coagulopathies and severe aortic stenosis.

Preoperative preparation had been performed for all patients including history, examinations, and investigations according to patient condition. In the preoperative holding area, a wide-pore IV cannula was inserted, and the patient was connected to standard monitors in the form of noninvasive arterial blood pressure, ECG, and pulse oximeter with measuring and recording of the baseline parameters. Ultrasound-guided ACB was performed for all patients in supine position under complete aseptic conditions. A high frequency $(6-15 \mathrm{MHz})$ linear probe of ultrasound machine (Sonosite Edge II) was positioned at the point midway between the anterior superior iliac spine and the upper pole of the patella. The adductor canal had been located as a hyperechoic structure beneath the sartorius muscle and after skin infiltration with $2 \mathrm{ml}$ of lidocaine $1 \%$, a spinal needle $22 \mathrm{G} \times 3.5$ inches was advanced with the guidance of ultrasound in an in-plane technique and bupivacaine $0.5 \% 15 \mathrm{ml}$ was injected.

In Group II patients, IPACK block was performed after ACB while the patient was in supine position with slight knee flexion (figure 1). Under complete aseptic conditions, the probe was applied to the popliteal fossa for identification of popliteal artery and femur. Then, the probe was slid distally for revealing the two femoral condyles (figure 2) followed by proximal sliding of the probe until the humps of the femoral condyles disappeared and the flat metaphysis appeared. After skin infiltration with $2 \mathrm{ml}$ of $1 \%$ lidocaine, a spinal needle $22 \mathrm{G} \times 3.5$ inches was advanced from the lateral aspect and directed across the space between the popliteal artery and femur and once the needle reached the medial edge of the femur, nearly at the level of the popliteal artery, negative aspiration was confirmed and bupivacaine $0.5 \% 15 \mathrm{ml}$ was injected incrementally as the needle was withdrawn (figure 3 ).
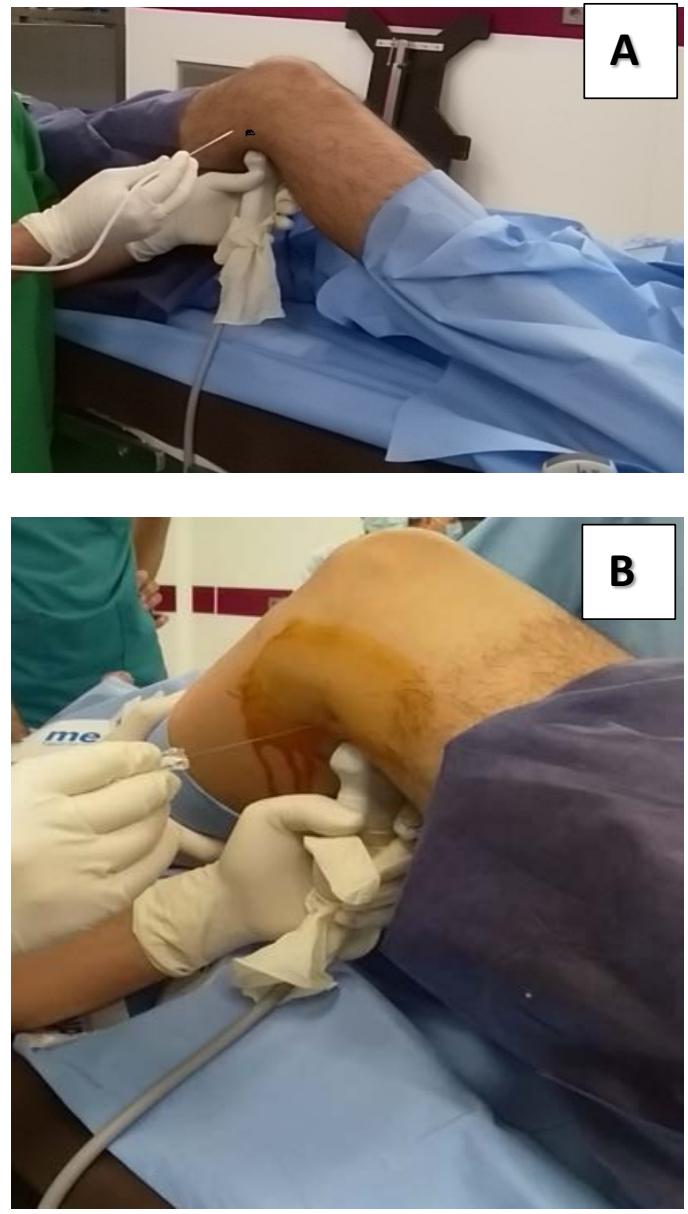

Fig 1: (Photos A and B) Probe position for IPACK block and needle direction. (Note: Holding the probe and the needle, black dot for the puncture site). 


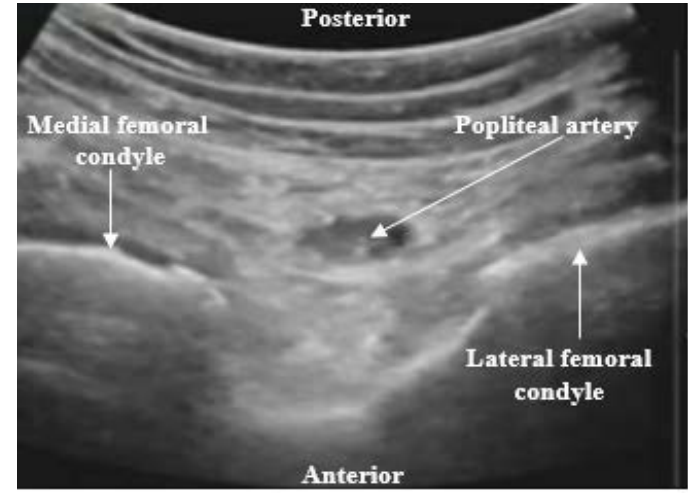

Fig 2: Two femoral condyles and popliteal artery
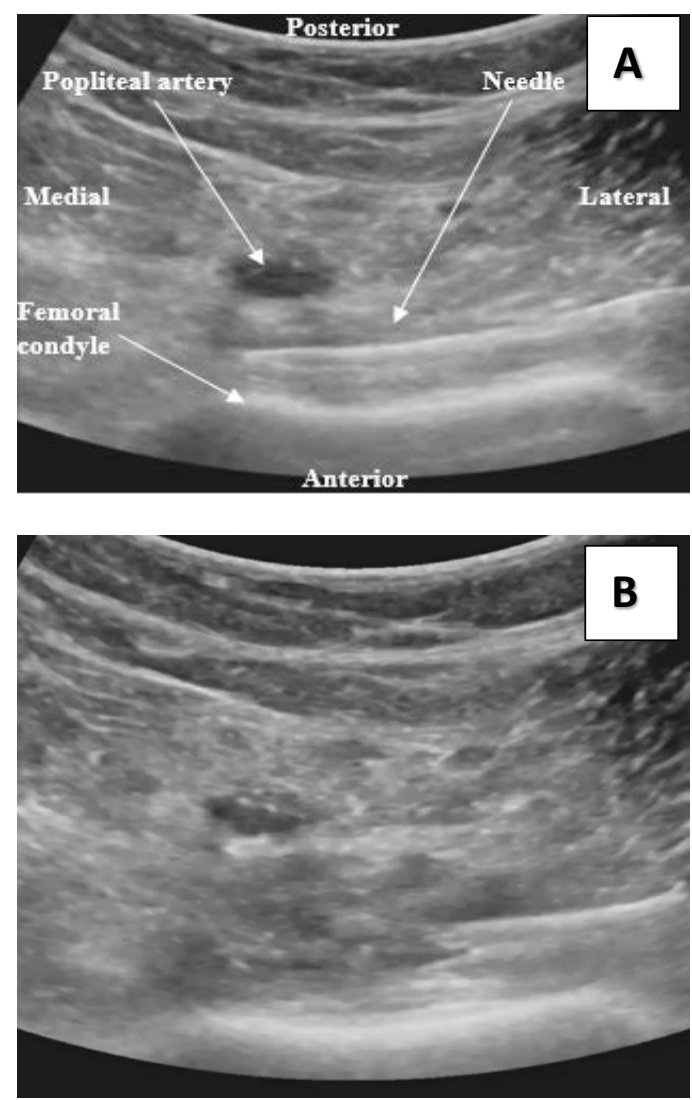

Fig 3: (Photos A and B). (A) Needle position during IPACK, (B) Injection during needle withdrawal.

After ACB for Group I patients or ACB plus IPACK for Group II, the patient was shifted to the operating room with continuation of patient's monitoring. After infusion of $10 \mathrm{ml} / \mathrm{kg}$ of lactated Ringer's solution within $15 \mathrm{~min}$, spinal anesthesia was given at the level of L3/4 while the patient was in the sitting position using a spinal needle 25G x 3.5 inches with injection of hyperbaric bupivacaine $0.5 \% 3 \mathrm{ml}$ under complete aseptic conditions. Intraoperative, heart rate, blood pressure, $\mathrm{SpO}_{2}$, and respiratory rate were measured and recorded every 5 minutes. After surgery, the patient was observed in post anesthesia care unit and he was shifted to the ward.

Visual analogue scale (VAS) score was assessed at 2, $4,8,12,16,24,32,40$ and $48 \mathrm{hrs}$ postoperative in the form of " horizontal line of 10 -cm long with two ends one end was labelled as no pain 0 and the other end was labelled as worst pain 10". The patient was asked to put a mark at the point which described pain intensity the best. The length of the line to the patient's mark is measured and recorded in centimeters. Total morphine consumption during postoperative $48 \mathrm{hrs}$, time to first rescue analgesia, and patient satisfaction were recorded. Rescue analgesia in the form IV morphine 3mg was given if VAS $\geq 4$. Perioperative complications were recorded and managed if occurred either opioid related as nausea and vomiting or related to the regional techniques used in the study as vascular injury and inadvertent intravascular injection of local anesthetic. Patient satisfaction with the analgesic regimen received was measured at the end of the assessment period using a scale from 0 to 10 where 0 meant extreme dissatisfaction while 10 meant extreme satisfaction. The patient was asked to circle 0 if no satisfaction and to circle any other number from 1 to 10 that best indicated the level of his satisfaction with pain management ${ }^{9}$.

\section{Sample size calculation}

Calculation of sample size was based on "MedCalc ${ }^{\circledR}$ version 12.3.0.0 program Ostend, Belgium"; statistical calculator based on $95 \%$ a confidence interval of $95 \%$ and power of the study $80 \%$ with $\alpha$ error $5 \%$. According to a previous study done by Sankineani et al. ${ }^{\mathbf{1 0}}$ showed that the Mean (SD) of VAS score at $8 \mathrm{hr}$ postoperative was decreased in patients received ACB plus IPACK $(1.43 \pm 0.64)$ compared with ACB alone $(2.91 \pm 0.64)$ with a $p$ value $<0.001$. So it can be relied upon in this study, based on this assumption, sample size was calculated according to these values produced a minimal sample size of 60 cases were enough to detect such a difference and subdivided into Group I $(n=30)$ and Group II ( $\mathrm{n}=30)$.

\section{Statistical analysis}

The analysis of data was performed by SPSS version 18 software package (SPSS Inc., Chicago, Ill., USA). Statistics were expressed in the form of mean \pm standard deviation (SD), median with interquartile range (IQR-median) for non-parametric data, and quantitative data were expressed as frequencies and percentage. The results had been analyzed by t-test, Mann Whitney, and Fisher's exact test. $P$-value was considered significant if $\leq 0.05$, nonsignificant if $>0.05$ is nonsignificant, and highly significant if $<0.001$.

\section{RESULTS}

As regards demographic data (age, sex, BMI, ASA, and duration of surgery), there were no statistically significant differences between the two groups (Table 1).

As regard to VAS, there were nonsignificant differences between both groups in the first 4 hours postoperative while at $8 \mathrm{hr}$ postoperative and up to $48 \mathrm{hr}$ postoperative, there were significant differences 
between both groups with less VAS scores in Group II (table 2).

Concerning total morphine consumption, it was less in Group II and the difference between both groups was statistically highly significant. Time to first rescue analgesia was shorter in Group I and the difference was highly significant. Patient satisfaction 48hr postoperative was higher in Group II than in

\begin{tabular}{lccc} 
& $\begin{array}{c}\text { Group I } \\
(\mathbf{n}=\mathbf{3 0})\end{array}$ & $\begin{array}{c}\text { Group II } \\
(\mathbf{n = 3 0 )}\end{array}$ & p-value \\
\hline Sex & $16(53.33 \%)$ & $15(50 \%)$ & \\
Female & $14(46.66 \%)$ & $15(50 \%)$ & 0.691 \\
Male & & & \\
Age (years) & $61.55 \pm 3.92$ & $62.96 \pm 4.15$ & 0.437 \\
Mean \pm SD & & & \\
Body mass index & $26.45 \pm 1.82$ & $27.35 \pm 1.64$ & 0.571 \\
Mean \pm SD & & & \\
ASA & $8(26.66 \%)$ & $7(23.33 \%)$ & 0.354 \\
I & $12(40.00 \%)$ & $13(43.33 \%)$ & \\
II & $10(33.33 \%)$ & $10(33.33 \%)$ & 0.746 \\
III & $134.63 \pm 37.75$ & $131.17 \pm 34.14$ & \\
Duration of surgery (min) & & & \\
\hline
\end{tabular}

Table 1: Comparison between group I and group II according to demographic data ( $\mathrm{p}$-value $>0.05$ is nonsignificant).

\begin{tabular}{|c|c|c|c|c|c|c|c|c|c|c|c|c|c|}
\hline \multirow{3}{*}{$\begin{array}{l}\text { Time } \\
2 \text { hrs }\end{array}$} & \multicolumn{4}{|c|}{ Group I } & \multicolumn{6}{|c|}{ Group II } & \multicolumn{3}{|r|}{ P-value } \\
\hline & \multicolumn{6}{|c|}{$(\mathrm{No}=30)$} & \multicolumn{6}{|c|}{$(\mathrm{No}=30)$} & \\
\hline & 0 & ( & 0 & - & 1 & ) & 0 & ( & 0 & - & 1 & ) & 0.869 \\
\hline 4 hrs & 2 & ( & 1 & - & 2 & ) & 1 & ( & 0 & - & 2 & ) & 0.677 \\
\hline 8 hrs & 2.5 & ( & 2 & - & 3 & ) & 1.5 & ( & 1 & - & 2 & ) & $<0.001$ \\
\hline 12 hrs & 3 & ( & 2 & - & 4 & ) & 2 & ( & 1 & - & 3 & ) & $<0.001$ \\
\hline 16 hrs & 3 & ( & 2 & - & 4 & ) & 2 & ( & 2 & - & 3 & ) & $<0.001$ \\
\hline 24 hrs & 3 & ( & 3 & - & 5 & ) & 3 & ( & 2 & - & 4 & ) & 0.005 \\
\hline 32 hrs & 4 & ( & 3 & - & 5 & ) & 3.5 & ( & 2 & - & 4 & ) & 0.042 \\
\hline 40 hrs & 4.5 & ( & 3 & - & 5 & ) & 3 & ( & 2 & - & 5 & ) & 0.018 \\
\hline 48 hrs & 3 & ( & 2 & - & 4 & ) & 2 & ( & 2 & - & 4 & ) & 0.013 \\
\hline
\end{tabular}

Table 2: Comparison between groups as regards postoperative VAS score. Data are expressed as Median (Inter Quartile Range).

\begin{tabular}{cccc}
\hline & $\begin{array}{c}\text { Group I } \\
(\mathrm{n}=30)\end{array}$ & $\begin{array}{c}\text { Group II } \\
(\mathrm{n}=30)\end{array}$ & p-value \\
\hline $\begin{array}{c}\text { Total morphine consumption (mg) } \\
\text { Mean } \pm \text { SD }\end{array}$ & $9.81 \pm 0.69$ & $6.54 \pm 0.73$ & $<0.001$ \\
$\begin{array}{c}\text { Time to first rescue analgesia (hours) } \\
\text { Mean } \pm \text { SD }\end{array}$ & $7.92 \pm 0.44$ & $9.73 \pm 0.63$ & $<0.001$ \\
$\begin{array}{c}\text { Patient satisfaction 48hr postoperative } \\
\text { (VRS) Median(IQR) }\end{array}$ & $6(5-7)$ & $8(7-9)$ & $<0.001$ \\
\hline
\end{tabular}


Table 3: Total morphine consumption, time to first rescue analgesia, and patient satisfaction. (p-value $<0.001$ highly significant, VRS: Verbal rating scale)

\section{DISCUSSION}

Achieving optimum postoperative analgesia with least side effects is a continuous important goal for anesthetists and postoperative care practitioners. Adductor canal block represents one of methods for postoperative analgesia after TKA, but it lacks the analgesic effect on posterior knee capsule which is innervated by terminal branches of the tibial nerve in addition to those of posterior branch of the obturator nerve. So, a supplemental analgesia is needed with $\mathrm{ACB}^{11}$. A relatively new technique, IPACK block, may represent a solution for the defect in analgesia of posterior knee after TKA using ACB alone ${ }^{12}$. Thus, proper pain management with peripheral nerve block can provide good analgesia, decrease the need for supplement analgesia, enhance early mobilization and rehabilitation, and shorten hospital stay ${ }^{\mathbf{8 , 1 3}}$.

In the present study, the patients received $\mathrm{ABC}$ plus IPACK in Group II showed less VAS scores 8 hours up to 48 hours postoperatively when compared to the patients received $\mathrm{ABC}$ alone in Group I. Coinciding with this study, Sankineani and his colleagues ${ }^{10}$ performed a prospective study involving 120 patients divided into two equal groups; one group received IPACK $(15 \mathrm{ml}$ of $0.2 \%$ ropivacaine plus ACB and the other group received ACB alone. They found that VAS scores were significantly lower in patients received IPACK plus ACB at 8hrs postoperative, 1 day postoperative, and 2 days postoperative with pvalue $<0.001$

Thobhani and his colleagues ${ }^{8}$ performed a study which included three groups, femoral nerve block (FNB) only, FNB with IPACK, and ACB with IPACK in patients subjected to TKA. Their study showed that ACB plus IPACK group produced adequate analgesia with VAS scores equivalent to that of the other two groups as the differences among the three groups were statistically nonsignificant with preservation of quadriceps strength which was affected by FNB. However, Opioid consumption was significantly less in FNB plus IPACK group in comparison to the other two groups. They concluded that the use of IPACK with FNB was associated with opioid sparing but this effect was not found when IPACK was used in combination with ACB.

Although sciatic nerve block when combined with $\mathrm{FNB}^{14}$ or $\mathrm{ACB}^{15}$ improves the analgesic effect and decreases both VAS scores and total morphine consumption after TKA, but it is associated with sensory and motor block distal to the knee that affect postoperative physiotherapy ${ }^{16}$.

Caballero-Lozada et al. ${ }^{17}$ performed a study in which 27 patients subjected to TKA under spinal anesthesia and received FNB plus IPACK for postoperative analgesia. They used Multimodal intravenous analgesia intraoperatively in the form of dipyrone $1 \mathrm{~g}$, diclofenac $75 \mathrm{mg}$, and dexamethasone $8 \mathrm{mg}$. Their study revealed that VAS scores was low (0,
$0.41 \pm 1.58,0.11 \pm 0.58,0.37 \pm 0.84$, and $0.78 \pm 1.25$ at 0 , $6,12,24$, and $48 \mathrm{hrs}$ postoperative respectively) and $73 \%$ of patients did not ask for rescue analgesia.

On contrary to the present study, Patterson et al. ${ }^{\mathbf{1 8}}$ performed a study to assess the analgesic effect of IPACK block with TKA. Their study involved two groups, one of them received continuous ACB with IPACK block and the other group received continuous ACB with subcutaneous normal saline injection as placebo at site of IPACK. Their results showed that using IPACK with ACB improved pain scores only in the immediate postoperative period and had no beneficial effect during subsequent pain assessment. Also, they found no difference in opioid consumption concluding that routine use of IPACK block with ACB in TKA may not have additional significant clinical benefit. They recommended that the indications for IPACK block may be applied where there are contraindications to the standard multimodal pain management (as those with contraindications to NSAIDs or acetaminophen), in case of chronic pain, or if the patient has opioid dependence.

\section{Limitations}

This study focused on the analgesic effect of IPACK block in combination with ACB regarding VAS and opioid analgesic requirements without evaluation of motor function possible benefits of the block in the form of early mobilization, early rehabilitation, and early patient discharge.

\section{CONCLUSION}

The combination of IPACK block with ACB has the potential of being an adequate technique for management of acute postoperative pain after TKA but this needs more researches of larger samples and use of other types of local anesthetics with different volumes and concentrations.

\section{REFERENCES}

1. Barrington JW, Halaszynski TM, and Sinatra RS. Perioperative pain management in hip and knee replacement surgery. Am J Orthop. 2014; 43 (4): 1-16.

2. Seo SS, Kim OG, Seo JH, et al. Comparison of the effect of continuous femoral nerve block and adductor canal block after primary total knee arthroplasty. Clin Orthop Surg. 2017; 9: 303-9.

3. Li JW, Ma YS, and Xiao LK. Postoperative Pain Management in Total Knee Arthroplasty. Orthop Surg. 2019; 11 (5): 755-61.

4. Memtsoudis SG, Yoo D, Stundner O, et al. Subsartorial adductor canal vs. femoral nerve block for analgesia after total knee replacement. Int Orthop. 2015; 39: $673 \quad-80$.

5. Gao F, Ma J, Sun W, et al. Adductor canal block versus femoral nerve block for analgesia after 
total knee arthroplasty: A systematic review and meta Eamalysisin. 2017; 33: 356-68.

6. Yadeau JT, Goytizolo EA, Padgett DE, et al. Analgesia after total knee replacement: local infiltration versus epidural combined with a femoral nerve blockade: a prospective, randomized pragmatic trial. Bone Joint J. 2013; 95-B (5): 629-35.

7. Baratta JL, Gandhi K, and Viscusi ER Perioperative pain management for total knee arthroplasty. J Surg Orthop Adv. 2014; 23 (1): 22-36.

8. Thobhani S, Scalercio L, Elliott CE, et al. Novel Regional Techniques for Total Knee Arthroplasty Promote Reduced Hospital Length of Stay: An Analysis of 106 Patients. Ochsner J. 2017; 17 (3): 233-8.

9. Subramanian B, Shastri N, Aziz L, et al. ASSIST - Patient satisfaction survey in postoperative pain management from Indian subcontinent. Journal of Anaesthesiology Clinical Pharmacology. 2017; 33 (1): 40-7.

10. Sankineani SR, Reddy ARC, Eachempati KK, et al. Comparison of adductor canal block and IPACK block (interspace between the popliteal artery and the capsule of the posterior knee) with adductor canal block alone after total knee arthroplasty: a prospective control trial on pain and knee function in immediate postoperative period. Eur J Orthop Surg Traumatol. 2018; 28(7): 1391-5.

11. Cong Z, Zhang L, and Ma F. The efficacy and safety of the infiltration of the interspace between the popliteal artery and the capsule of the knee block in total knee arthroplasty: A prospective randomized trial protocol. Medicine (Baltimore). 2020; 99 (33): 21670.

12. Surjya Prasad U, Sudhakar T, Sunil K, et al. Postoperative Analgesia in Total Knee Arthroplasty (TKA) - The Changing Trends. Biomed J Sci \& Tech Res. 2017; 1(3): 713-9.

13. El-Emam EM and Abd El motlb EA. UltrasoundGuided Adductor Canal Block versus Combined Adductor Canal and Infiltration between the Popliteal Artery and the Posterior Capsule of the Knee Block for Osteoarthritis Knee Pain. Anesthesia: Essays and Researches. 2020; 14(1): 127-31.

14. Abdallah FW, Madjdpour C, and Brull R. Is sciatic nerve block advantageous when combined with femoral nerve block for postoperative analgesia following total knee arthroplasty? A meta-analysis. Can J Anaesth. 2016; 63 (5): 55268.

15. Eldegwy HM and Negm MA. Adductor Canal Block Combined with Sciatic Nerve Block Vs. Local Analgesic Infiltration for Pain Control after Total Knee Arthroplasty. J Anesth Clin Res. 2018; 9: 799-805.

16. Sinha SK, Abrams JH, Arumugam S, et al. Femoral nerve block with selective tibial nerve block provides effective analgesia without foot drop after total knee arthroplasty: a prospective, randomized, observer-blinded study. Anesth Analg. 2012; 115 (1): 202-6.

17. Caballero-Lozada AF, Gomez JM, Ramírez JA, et al. IPACK block: emerging complementary analgesic technique for total knee arthroplasty. Colombian Journal of Anesthesiology. 2020; 48 (2): 78-84.

18. Patterson ME, Vitter J, Bland K, et al. The Effect of the IPACK Block on Pain After Primary TKA: A Double-Blinded, Prospective, Randomized Trial. The Journal of Arthroplasty. 2020; 35 (6): $1-5$. 